\title{
Predictive Simulation of Bidirectional Glenn Shunt Using a Hybrid Blood Vessel Model
}

\author{
Hao $\mathrm{Li}^{1,2}$, Wee Kheng Leow ${ }^{1,2}$, and Ing-Sh Chiu ${ }^{3}$ \\ 1 Dept. of Computer Science, National University of Singapore, Singapore \\ 2 Image \& Pervasive Access Lab (IPAL), UMI CNRS Singapore \\ 3 Dept. of Surgery, National Taiwan University Hospital, Taipei, Taiwan \\ \{lihao, leowwk\}@comp.nus.edu.sg, ingsh@ntu.edu.tw
}

\begin{abstract}
This paper proposes a method for performing predictive simulation of cardiac surgery. It applies a hybrid approach to model the deformation of blood vessels. The hybrid blood vessel model consists of a reference Cosserat rod and a surface mesh. The reference Cosserat rod models the blood vessel's global bending, stretching, twisting and shearing in a physically correct manner, and the surface mesh models the surface details of the blood vessel. In this way, the deformation of blood vessels can be computed efficiently and accurately. Our predictive simulation system can produce complex surgical results given a small amount of user inputs. It allows the surgeon to easily explore various surgical options and evaluate them. Tests of the system using bidirectional Glenn shunt (BDG) as an application example show that the results produced by the system are similar to real surgical results.
\end{abstract}

\section{Introduction}

Many cardiac surgeries for correcting congenital heart defects, such as arterial switch operations and bidirectional Glenn shunt [1], involve complex operations on the cardiac blood vessels. At present, cardiac surgeons mostly rely on echocardiography, cardiac catheterization and CT images of a patient's heart to examine the specific anatomical anomalies of the patient. Without appropriate surgical planning and visualization tool, they often resort to manual drawings to visualize the surgical procedures and the expected surgical results. This approach is imprecise and is impossible to provide details of the possible outcome of the surgical procedures. To improve the precision and effectiveness of cardiac surgery planning, novel surgical simulation systems are desired.

Among existing surgical simulation systems, reactive systems (e.g., 223]) attempt to simulate real-time displacement and deformation of body tissues in response to user inputs that emulate surgical operations. They are useful for training and navigational planning of surgical operations. However, to use a reactive system to predict results of complex surgeries, the surgeon would need to go through all the detailed surgical steps, which is tedious and time-consuming.

In contrast, predictive simulation systems (e.g., 4]) aim at efficiently producing complex surgical results based on the physical properties of the anatomies 
and a small amount of user inputs that indicate the surgical options. In this way, the surgeon can easily explore various surgical options and evaluate the predicted surgical results without going through the surgical details.

This paper illustrates a predictive simulation system for bidirectional Glenn shunt (BDG), which is a very important cardiac surgery for the treatment of several congenital heart defects (Sec. 3). In the predictive simulation of BDG, as in the simulation of other surgical procedures that involve soft tissues, the model objects should deform according to their physical properties. In addition, the deformation of the blood vessels should comply with an additional constraint: they should not be overly stretched or twisted to avoid obstruction of blood flow. This constraint translates to the requirement of modeling global strains such as bending, stretching and twisting of the blood vessels.

Blood vessel deformation can be modeled using 3D finite elements [5] or thin shell models [67]. These methods are in general computationally expensive. For reactive simulation, mass spring model has been applied to the simulation of cardiac tissue and blood vessel deformation [2]3. However, its simulation accuracy highly depends on careful placements of springs between mass points, which may not have physical correlates. More importantly, these general surface/volume deformation methods do not explicitly model global bending and twisting, which are important characteristics of blood vessels.

To meet the requirement of modeling global strains in the predictive simulation of BDG, this paper presents a hybrid approach for modeling blood vessel deformation (Sec. 2). The hybrid blood vessel model binds a surface mesh elastically to a reference Cosserat rod [8]. The mesh model represents the surface details of the blood vessel while the reference rod models global bending, stretching, twisting and shearing of the vessel. Deformation of the hybrid model is accomplished by first deforming the reference rod according to Cosserat theory, then deforming the mesh according to its binding to the rod and its surface elastic energy. This approach allows the blood vessel to deform in a physically correct manner in relation to its global strains. It also allows the surface to deform realistically and efficiently. Moreover, the hybrid model provides structural information of the blood vessel, thus reducing the amount of user inputs that indicate surgical options (Sec. 3). Experimental results (Sec.4) confirm the effectiveness of our model in the predictive simulation of BDG. Note that although the example application in this paper is BDG, our blood vessel model is general enough for the predictive simulation of other complex cardiac surgeries.

In comparison to our work, existing reactive simulation systems of cardiac surgery [23] focus on low-level operations such as incision and retraction of the heart [23] and suturing of ventricular septal defect [2, rather than complex operations on the cardiac blood vessels. For predictive simulation of cardiac surgery, Li et al. 4] applied differential geometry approach to predict the surgical results of aorta reconstruction. The predictive simulation idea is in spirit similar to that of this paper. However, the deformation approach adopted in [4] is a pure geometrical approach, which is not necessarily accurate. Moreover, it took into consideration only the surface elastic energies and did not model the 
global strains of blood vessels. Although torsion energy can be approximated as described in [9], it is nontrivial to incorporate the torsion energy term to affect the behavior of the blood vessel model.

\section{Hybrid Blood Vessel Model}

Our hybrid model of a blood vessel consists of a reference Cosserat rod and a surface mesh of the blood vessel (Fig. 2.1). For a given surface mesh of a blood vessel, we construct the hybrid model by fitting a reference rod to the centerline of the surface using the technique described in 910. A binding relationship between the surface model and the reference rod is then established. The deformation of the blood vessel is achieved by first deforming the reference rod according to Cosserat theory 8] (Sec. 2.1), then deforming the mesh model according to the surface elastic energy in terms of surface bending and stretching, and the established binding relationship to the reference rod (Sec. 2.2).

\subsection{Cosserat Rod}

A Cosserat rod $C[8$ is represented by a 3D curve $\mathbf{r}(s)$ and orthonormal directors $\mathbf{d}_{k}(s)$, with $k \in\{1,2,3\}$ and the arc length parameter $s \in[0, L] . \mathbf{d}_{3}(s)$ is normal to the cross-sectional plane $\mathcal{X}_{s}$ of the rod at $s . \mathbf{d}_{1}(s)$ and $\mathbf{d}_{2}(s)$ are in $\mathcal{X}_{s}$ and point at two material points on the rod's surface (Fig. 2.1).

The strains of a Cosserat rod are expressed as linear strain vector $\boldsymbol{v}(s)$ and angular strain vector $\boldsymbol{u}(s)$ [8] such that

$$
\boldsymbol{v}(s)=\partial_{s} \mathbf{r}(s), \quad \partial_{s} \mathbf{d}_{k}(s)=\boldsymbol{u}(s) \times \mathbf{d}_{k}(s), \quad k \in\{1,2,3\} .
$$

The strain vectors $\boldsymbol{u}(s)$ and $\boldsymbol{v}(s)$ can be resolved into three components by the directors $\mathbf{d}_{k}$ to yield the strain variables $u_{k}$ and $v_{k}$ :

$$
u_{k}=\boldsymbol{u} \cdot \mathbf{d}_{k}, \quad v_{k}=\boldsymbol{v} \cdot \mathbf{d}_{k}, \quad k \in\{1,2,3\} .
$$

Together with global translation and rotation of the rod, $u_{k}$ and $v_{k}$ define the rod's configuration, i.e., $\mathbf{r}$ and $\mathbf{d}_{k}$. The components $u_{1}$ and $u_{2}$ are the curvatures along $\mathbf{d}_{1}$ and $\mathbf{d}_{2}$ which measure the bending of the rod, while $u_{3}$ measures twisting. The components $v_{1}$ and $v_{2}$ measure shear, and $v_{3}$ measures stretching. Let us denote

$$
\mathbf{u} \equiv\left[u_{1}, u_{2}, u_{3}\right]^{\top}, \quad \mathbf{v} \equiv\left[v_{1}, v_{2}, v_{3}\right]^{\top} .
$$

Assuming the Kirchhoff constitutive relations, the couple (i.e., inner torque) $\mathbf{m}(s)$ and stress $\mathbf{n}(s)$ experienced by the rod are

$$
\mathbf{m}(s)=\mathbf{J}(s)\left(\mathbf{u}(s)-\mathbf{u}^{0}(s)\right), \quad \mathbf{n}(s)=\mathbf{K}(s)\left(\mathbf{v}(s)-\mathbf{v}^{0}(s)\right),
$$

where $\mathbf{J}(s)$ and $\mathbf{K}(s)$ are stiffness matrices that depend on the geometric moments of inertia and material properties of the rod, and $\mathbf{u}^{0}(s)$ and $\mathbf{v}^{0}(s)$ are the strain values in the initial configuration. The potential energy of the rod is [8]:

$$
\mathcal{E}=\frac{1}{2} \int_{0}^{L}\left[\left(\mathbf{u}(s)-\mathbf{u}^{0}(s)\right)^{\top} \mathbf{m}(s)+\left(\mathbf{v}(s)-\mathbf{v}^{0}(s)\right)^{\top} \mathbf{n}(s)\right] d s .
$$




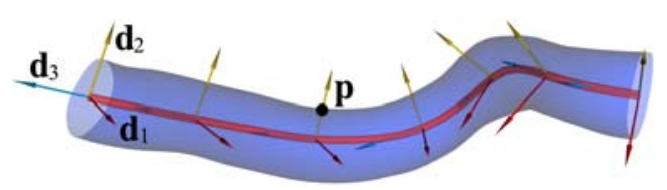

Fig. 1. A hybrid blood vessel model with its reference rod, directors and surface mesh

Given boundary conditions on the positions $\mathbf{r}$ or the directors $\mathbf{d}_{k}$, the deformed configuration $C^{t}=\left\{\mathbf{r}^{t}, \mathbf{d}_{k}^{t}\right\}$ is the equilibrium state of the rod, with minimum potential energy $\mathcal{E}$ that minimizes the difference between the strains.

\subsection{Blood Vessel Surface Deformation}

Binding Surface to Cosserat Rod. If the cross-sections do not change, the surface of a blood vessel can be considered as a shape function defined by the reference Cosserat rod. For each mesh vertex $\mathbf{p}$, there exists an $s$ such that $\mathbf{p}$ is on the cross-section $\mathcal{X}_{s}$ of the blood vessel at $s$. A one-to-one mapping function f can then be established between the local coordinates $(x, y, z)$ in the directors $\mathbf{d}_{k}$ at $s$ and the global coordinates $\mathbf{p}$ :

$$
\mathbf{p}=\mathbf{f}(s, x, y, z)=\mathbf{r}(s)+x \mathbf{d}_{1}(s)+y \mathbf{d}_{2}(s)+z \mathbf{d}_{3}(s) .
$$

In the initial configuration, the local coordinates $(x, y, z)$ for each mesh vertex are computed from the initial reference rod. When the reference Cosserat rod deforms, function $\mathbf{f}$ defines the reference binding position $\mathbf{p}^{\prime}$ of the mesh vertex $\mathbf{p}$ in the deformed configuration. The elastic binding energy between the surface mesh and the reference Cosserat rod can then be defined as:

$$
E_{c}(\mathbf{p})=k_{c}\left\|\mathbf{p}-\mathbf{p}^{\prime}\right\|_{2}^{2}
$$

where $k_{c}$ is the corresponding binding coefficient.

Surface Bending and Stretching. When a blood vessel deforms, its surface undergoes stretching and bending, which incur stretching and bending energies. For discrete meshes, various techniques have been presented to approximate the energies (e.g., [7). Since stretching and bending are essentially non-linear characteristics, the resulting deformable model are usually non-linear, which may be computationally costly.

One efficient way to approximate the bending energy is to use Laplacian operators 1112. Since Laplacian is a linear operator, it is rotation variant. Traditional ways of achieving rotation-invariant Laplacian is to approximate the rotation of Laplacian from known handle vertices [12] or by using multi-resolution hierarchies [11]. In our model, rotation-invariance is achieved by measuring the Laplacians with respect to the directors of the reference rod, which are intrinsic properties of the rod. This is a more natural approach to achieving rotation invariance of Laplacian for tubular objects like blood vessels. Therefore, the reference Cosserat rod serves the important roles of modeling global strains of 


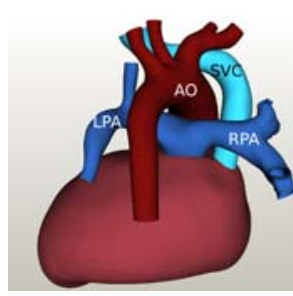

(a)

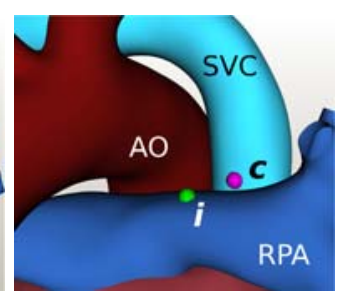

(b)

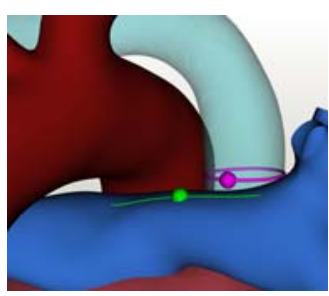

(c)

Fig. 2. Models and inputs. (a) Heart and blood vessel models reconstructed from a patient's CT images. (b) User input points to indicate surgical options. (c) Crosssectional cut path and incision path generated by the system.

the blood vessel, as well as achieving rotation-invariant Laplacians. The surface bending energy at a vertex $\mathbf{p}$ with its Laplacian $\mathbf{l}(\mathbf{p})$ is:

$$
E_{b}(\mathbf{p})=k_{b}\left\|\mathbf{l}(\mathbf{p})-\mathbf{R}(\mathbf{p}) \mathbf{l}^{0}(\mathbf{p})\right\|_{2}^{2},
$$

where $\mathbf{l}^{0}(\mathbf{p})$ is the Laplacian of $\mathbf{p}$ in the initial configuration, $\mathbf{R}(\mathbf{p})$ is the rotation of the corresponding directors, and $k_{b}$ is the bending stiffness.

Surface stretching energy is measured by the change of edge lengths similar to [7. For an edge $\mathbf{e}$ that connects vertices $\mathbf{p}_{i}$ and $\mathbf{p}_{j}$, the stretching energy is:

$$
E_{s}(\mathbf{e})=k_{s}\left(\left\|\mathbf{p}_{i}-\mathbf{p}_{j}\right\|-\left\|\mathbf{p}_{i}^{0}-\mathbf{p}_{j}^{0}\right\|\right)^{2},
$$

where $k_{s}$ is the stretching stiffness related to the Young's modulus, and $\left\|\mathbf{p}_{i}^{0}-\mathbf{p}_{j}^{0}\right\|$ is the edge length in the initial configuration.

The total surface deformation energy to be minimized is thus:

$$
E=E_{c}+E_{b}+E_{s} .
$$

Note that the binding and bending terms are both linear. The stretching term, although non-linear, can be solved efficiently in an iterative way described in [13. Hence, the overall surface deformation can be achieved efficiently.

\section{Predictive Simulation of Bidirectional Glenn Shunt}

Bidirectional Glenn shunt (BDG) is a very important operation involved in the treatment of several congenital heart detects such as tricuspid atresia, hypoplastic left heart syndrome, and single ventricle anomalies [1. It is performed by first detaching the superior vena cava (SVC) from the heart through a cross-sectional cut on the SVC, then making an incision on the top side of the right pulmonary artery (RPA), and connecting the distal end of the SVC to the top side of RPA. After BDG, venous blood from the head and upper limbs will pass directly to the lungs, bypassing the right ventricle such that the volume load on the ventricle will be decreased and the oxygen saturation will be improved. 
In the surgical planning of BDG, the surgeon needs to decide: a) the position of the cross-sectional cut on the SVC to detach it from the heart; b) the incision path on the top side of the RPA; c) the correspondence between the two joining boundaries to join the SVC and RPA with minimum twisting.

These decisions should be carefully made for every patient to reduce the potential risk (e.g., obstruction of blood flow) and to improve the long-term outcome. In addition, the surrounding cardiac structures (e.g., the aorta and the heart) limit the desirable deformation of the SVC and RPA, making the surgical decisions very crucial.

\subsection{Predictive Simulation Algorithm}

Our predictive simulation system allows the user to simply pick a point on the SVC and RPA respectively to indicate the surgical decision (Fig. 2(b)). It then automatically predicts the result of BDG with minimum blood vessel deformation. The simulation algorithm consists of the following three steps:

Step 1. Given the cut position c on the SVC (Fig. 2(b)), the algorithm computes its projection to the reference rod of SVC to get the parameter $s$ and the crosssectional plane $\mathcal{X}_{s}$ of the reference rod (Fig. 2(c)). The surface is cut by this cross-sectional plane and the reference rod is split into two parts.

Step 2. Given the incision point $\mathbf{i}$ on the RPA (Fig. 2(b)), an incision path is drawn on RPA's surface (Fig. 2(c)) such that it is parallel to RPA's reference rod and the mid-point of the path is the input point $\mathbf{i}$. The length of the incision path is determined by the perimeter of the distal end of the cut SVC.

Step 3. The point correspondence between the two joining boundaries is established such that the center of the distal end of SVC is aligned with point $\mathbf{i}$, and the director $\mathbf{d}_{3}$ is normal to the surface of RPA. Next, the deformation algorithm is applied to solve for the final configuration that minimizes the Cosserat rod energy $\mathcal{E}$ (Eq. [5), the surface energy $E$ (Eq. [10), and an additional spring energy introduced to join the corresponding points of the two boundaries.

The final configuration illustrates the expected surgical results based on the given surgical decision, which is the minimally deformed configuration. In addition, the system also illustrates a way of cutting and joining the blood vessels with minimum deformation.

\section{Tests and Discussions}

For testing our hybrid model, a straight tube (Fig. 3(a)) was constructed to model a real elastic tube that the surgeon uses to emulate blood vessels during surgery planning. Figure 3 (b, c) compares the bending and twisting deformation of the model and that of the real tube, which was manipulated by hand at its two ends. The similarity of our tube model to the real tube shows the correctness of our hybrid approach in simulating tubular object deformation. Figure 3 (d) 


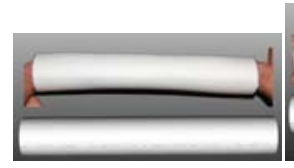

(a)

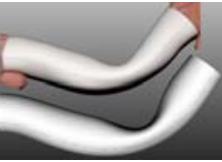

(b)

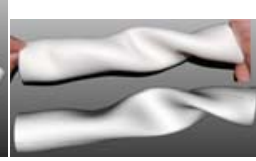

(c)

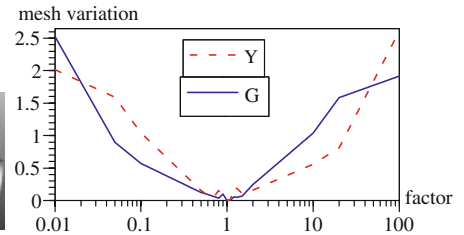

(d)

Fig. 3. Deformation of hybrid model (lower) in comparison with real elastic tube manipulated by hand (upper). (a) Initial configuration, (b) bending, (c) twisting. (d) Mesh variation with respect to change of Young's $(Y)$ and shear $(G)$ moduli.

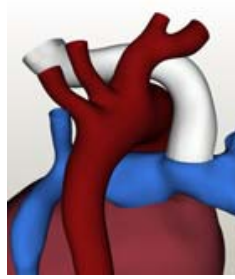

(a)

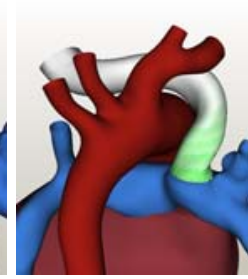

(b)

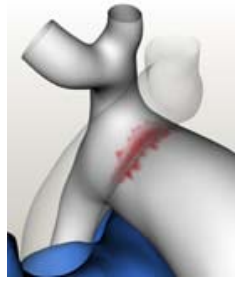

(c)

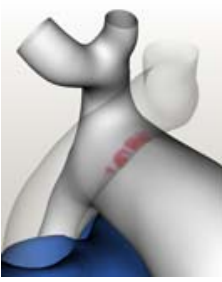

(d)

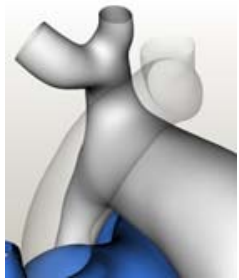

(e)

Fig. 4. Predicted surgical results of various surgical options. (a) Desired result, free of congestion and twisting. (b) Result with undesirable twisting indicated in green. (c-e) Different selections of cut points on SVC result in different amount of pressure due to collision with aorta. SVC's are made semi-transparent to illustrate the pressure in red.

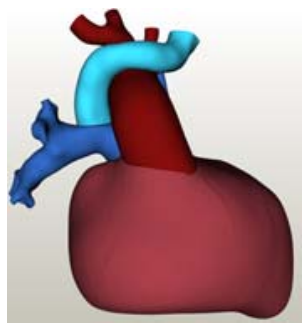

(a)

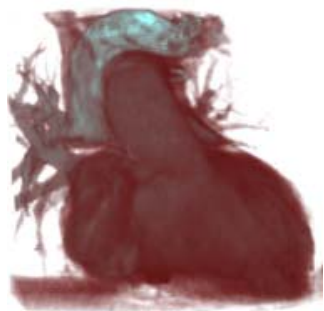

(b)

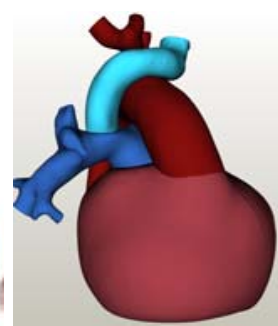

(c)

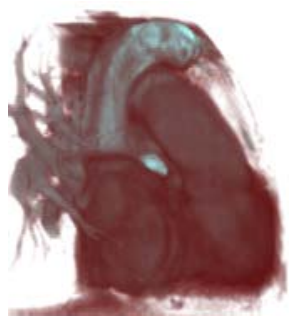

(d)

Fig. 5. Comparison of (a, c) simulation results with (b, d) volume rendering of postoperative CT data. (a, b) Frontal view. (c, d) Side view.

illustrates mesh variation with respect to change of material property in simulating the twisting example (Fig. 3)(c)). The Young's and shear moduli varied from their default values with a factor ranging from 0.01 to 100 . Figure 3(d) shows that the deformation algorithm is robust, i.e., produces small mesh variation with small change of material property. The slight fluctuation of the curves for factors close to 1 may be attributed to the non-linear behavior of the model and numerical error in discrete optimization. 
For testing the predictive simulation algorithm, 3D mesh models of the heart, aorta, pulmonary artery and superior vena cava were segmented and reconstructed from a patient's CT images (Fig. 2(a)), and the hybrid models were constructed. For blood vessels with branches such as the aorta and pulmonary trunk, the major branches can also be modeled using the hybrid model with reference rods connected to the reference rod of the main trunk, thus constructing a tree structure of rods. The BDG simulation algorithm was performed on the blood vessel models to join the SVC to the RPA. Various surgical options were tested as inputs for evaluating the predicted surgical results.

Figure 4(a) shows a desired surgical result which is free of congestion and twisting. Explicit modeling of global strains in our model allows strain values to be easily visualized. For example, by intentionally rotating the distal end of SVC, undesirable twisting of SVC was produced and visualized (Fig. 4 (b)). The selection of the cut position on the SVC is crucial since it affects the length of the cut SVC. As shown in Figure 4(c-e), when the cut SVC was too short, it collided with the aorta thus resulting in pressure on both vessels (Fig. 4(c)). The pressure decreased with increasing SVC length (Fig. 4(d)), and became zero when the cut SVC was long enough to avoid collision (Fig. 4(e)).

Note that quantitative validation of the predictive simulation system is difficult to achieve because the reconstructed 3D models cannot be exact due to errors in CT scanning and image segmentation. Moreover, the blood vessel shape is affected by other factors such as blood pressure. Therefore, this paper presents only a qualitative validation by comparing the simulation result with the volume data of the patient's postoperative CT rendered by VTK 14. Frontal and side views of the volume rendering were selected such that the SVC and aorta can be clearly seen. Figure 5 shows that the shapes of the blood vessels in our simulation result are similar to those in the volume data. The heart model differs somewhat from the real heart due to segmentation error and rendering effects.

The above tests were carried out on a $2.33 \mathrm{GHz}$ Core 2 Duo PC. The execution time for BDG simulation is on average 10 seconds, with the blood vessel models containing a total of 24, 393 vertices (AO: 8, 995, PA: 9, 824, VC: 5, 574).

\section{Conclusion}

This paper presented a predictive simulation system for bidirectional Glenn shunt (BDG) in cardiac surgery. The system uses a hybrid approach to model blood vessel deformation. The hybrid model binds a reference Cosserat rod and a surface mesh elastically. The reference rod models the global strains of the blood vessel in a physically correct manner, while the 3D mesh models the surface details of the blood vessel. In this way, blood vessel deformation can be achieved accurately and efficiently. Our blood vessel model also provides structural information that facilitates predictive simulation procedure. Experiments and qualitative validation confirm the feasibility and effectiveness of the hybrid blood vessel model for the predictive simulation of BDG. 


\section{References}

1. Gardner, T.J., Spray, T.L.: Operative Cardiac Surgery, 5th edn. Arnold (2004)

2. Sørensen, T.S., Greil, G.F., Hansen, O.K., Mosegaard, J.: Surgical simulation - a new tool to evaluate surgical incisions in congenital heart disease? Interact. CardioVasc. Thorac. Surg. 5(5), 536-539 (2006)

3. Mosegaard, J.: LR-spring mass model for cardiac surgical simulation. In: Proc. MMVR, pp. 256-258 (2004)

4. Li, H., Leow, W.K., Chiu, I.S.: Modeling torsion of blood vessels in surgical simulation and planning. In: Proc. MMVR, pp. 153-158 (2009)

5. Nealen, A., Müller, M., Keiser, R., Boxermann, E., Carlson, M.: Physically based deformable models in computer graphics. In: Proc. Eurographics, pp. 71-94 (2005)

6. Terzopoulos, D., Platt, J., Barr, A., Fleischer, K.: Elastically deformable models. In: Proc. ACM SIGGRAPH, pp. 205-214 (1987)

7. Grinspun, E.: A discrete model of thin shells. In: SIGGRAPH Courses (2006)

8. Antman, S.S.: Nonlinear Problems of Elasticity. Springer, Heidelberg (1995)

9. Li, H., Leow, W.K., Qi, Y., Chiu, I.S.: Predictive surgical simulation of aorta reconstruction in cardiac surgery. In: Proc. MMVR, pp. 159-161 (2009)

10. Kirchberg, K.J., Wimmer, A., Lorenz, C.H.: Modeling the human aorta for MRdriven real-time virtual endoscopy. In: Proc. MICCAI, pp. 470-477 (2006)

11. Botsch, M., Sorkine, O.: On linear variational surface deformation methods. IEEE Trans. on Visualization and Computer Graphics 14(1), 213-230 (2008)

12. Masuda, H., Yoshioka, Y., Furukawa, Y.: Interactive mesh deformation using equality-constrained least squares. Computers \& Graphics 30(6), 936-946 (2006)

13. Weng, Y., Xu, W., Wu, Y., Zhou, K., Guo, B.: 2D shape deformation using nonlinear least squares optimization. The Visual Computer 22(9), 653-660 (2006)

14. Kitware, Inc.: The Visualization Toolkit, http://www.vtk.org 